\title{
Pre-transplantation and post-transplantation serum bone alkaline phosphatase levels in renal transplant patients
}

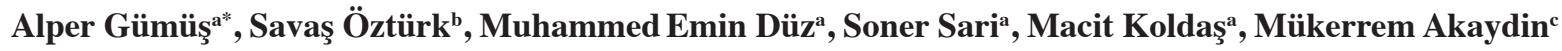 \\ ${ }^{a}$ Department of Medical Biochemistry, Haseki Training and Research Hospital, İstanbul, Turkey \\ ${ }^{b}$ Department of Nephrology, Haseki Training and Research Hospital, İstanbul, Turkey \\ ${ }^{c}$ Beckman Coulter Istanbul Office, Istanbul, Turkey
}

\section{ARTICLE INFO}

\section{Article History}

$\begin{array}{ll}\text { Received } & 03 / 05 / 2014 \\ \text { Accepted } & 12 / 06 / 2014\end{array}$

\section{* Correspondence to: \\ Alper Gümüş \\ Department of Medical Biochemistry, Haseki Training and Research Hospital, İstanbul, Turkey \\ e-mail: dralpergumus@gmail.com}

\section{Keywords:}

Bone alkaline phosphatase

End stage renal disease

Renal osteopathology

Renal transplantation

\section{ABSTRACT}

Bone Alkaline Phosphatase (BAP) reflects the biosynthetic activity of the osteoblasts. End-stage renal disease and renal transplantation have effects on bone metabolism. The aim of this study was to evaluate serum BAP levels in pre-transplantation (pre-tx) and post-transplantation (post-tx) samples obtained from patients who underwent a renal transplantation. Forty end-stage renal disease patients ( 16 male and 24 female) undergoing transplantation and 40 healthy individuals (20 female and 20 male) volunteered for this study. The serum samples were obtained before the onset of immunosuppressive treatment and two months after renal transplantation. Serum BAP levels were measured by Ostease Kit (Beckman Coulter, California, USA) at DXI 800 immunoassay autoanalyzer. There were significant differences between control group and pre-tx group $(\mathrm{p}=0.015)$ and between control group and post-x group $(\mathrm{P}<0.001)$. The serum BAP levels were lower in the post-tx group than that of the control group the control group, but this difference was not statistically significant $(\mathrm{p}=0.023)$. These results suggest that osteoblast activity is suppressed during chronic renal failure and transplantation worsens osteoblastic inactivity, thus causing to enter a non-dynamic state the bone. BAP may be a useful parameter for assessing the metabolic status of bone before and after renal transplantation.

J. Exp.Clin. Med., 2014; 31:91-93

\section{Introduction}

Renal osteopathology is the inevitable result of end-stage renal disease (ESRD). While transplantation is capable of resolving many complications of ESRD, occasionally, a disturbance to bone mineral metabolism persists. Bone disease after transplantation is a persistent form of osteopathology. Secondary hyperparathyroidism, aluminum osteomalacia and postoperative glucocorticoid therapy have been proposed as causes of this osteopathology (Heaf, 2003). Regardless of the primary cause, monitoring the metabolic status of a patient's bone throughout the pre-transplantation (pre-tx) and post-transplantation (post-tx) period is important. Bone alkaline phosphatase (BAP) is an alkaline phosphatase (ALP) isoenzyme (EC 3.1.3.1). ALP is a hydrolase enzyme responsible for removing phosphate groups from many types of molecules. Different ALP isoenzymes have been identified and are encoded by different genes. BAP is an isoform of the tissue non-specific ALP gene (Weiss and Ray, 1988). For the studies that evaluate bone turnover, BAP is one of the most commonly measured parameters. The serum BAP level reflects the metabolic status of osteoblasts (Sardiwal and Gardham, 2012). The aim of this study was to evaluate BAP levels in pre-tx and post-tx samples obtained from the patients.

\section{Materials and methods}

Forty chronic ESRD patients (16 male and 24 female) undergoing transplantation and 40 healthy individuals (20 female and 20 male) volunteer were included in this study. Experiments were undertaken with the understanding 


\begin{tabular}{|c|c|c|c|c|c|c|}
\hline & & MEAN & MIN & MAX & SD & SE \\
\hline \multirow[t]{2}{*}{ Age } & patient & 37.2 & 8 & 58 & 11.8 & \\
\hline & control & 35.6 & 10 & 60 & 12.4 & \\
\hline \multirow[t]{2}{*}{ Weight (kg) } & patient & 64.3 & 17.4 & 95.3 & 15.9 & \\
\hline & control & 75.4 & 32.6 & 101 & 21 & \\
\hline \multirow[t]{2}{*}{ Height $(\mathbf{c m})$} & patient & 162 & 117 & 181 & 14 & \\
\hline & control & 165 & 135 & 185 & 16 & \\
\hline \multicolumn{2}{|c|}{ Duration of dialysis (month) } & 17.2 & 1 & 96 & 20.9 & \\
\hline \multicolumn{2}{|c|}{ Pre-tx Ca (mg/dL) } & 9.56 & 7.1 & 12 & 0.97 & 0.15 \\
\hline \multicolumn{2}{|c|}{ Post-tx Ca (mg/dl) } & 8.9 & 5 & 11 & 1.16 & 0.22 \\
\hline \multicolumn{2}{|c|}{ Pre-tx P (mg/dL) } & 5.27 & 2.5 & 8.2 & 1.45 & 0.22 \\
\hline \multicolumn{2}{|c|}{ Post-tx (mg/dL) } & 3.29 & 1 & 6 & 1.14 & 0.18 \\
\hline \multicolumn{2}{|c|}{ Control group BAP $(\mu \mathrm{g} / \mathrm{L})$} & 17.07 & 7.75 & 68.91 & 9.95 & 1.57 \\
\hline \multicolumn{2}{|c|}{ Pro-tx BAP $(\mu \mathrm{g} / \mathrm{L})$} & 5.19 & 1.04 & 41.7 & 7.65 & 1.21 \\
\hline \multicolumn{2}{|c|}{ Post-tx BAP $(\mu \mathrm{g} / \mathrm{L})$} & 2.37 & 0.24 & 8.68 & 1.5 & 0.23 \\
\hline
\end{tabular}

MIN: Minimum; MAX: Maximum; SD: Standart deviation; SE: Standart error

and written consent of each subject. Demographic data for the patient and control groups are shown in Table 1.

This prospective study was conducted with patients who underwent renal transplantation from living, related donors from January 2012 to March 2012. Co-morbidities that may have influenced bone turnover included chronic liver disease, a prior history of renal transplantation and malignancy; thus, patients with any of those co-morbidities were excluded from the study. All donor recipients received immunosuppressive therapy that consisted of corticosteroids, tacrolimus/sirolimus and mycofenolate mofetil. Patients who were given sirolimus therapy and those with a high mismatch number were given anti-CD25 induction therapy (basiliximab or daclizumab).

The serum samples were obtained before the onset of immunosuppressive treatment and two months after renal transplantation and were stored at $-50^{\circ} \mathrm{C}$. BAP levels were measured using an Ostease Kit (catalog number: 37300) (Beckman Coulter, California,USA) at DXI 800 immunoassay auto-analyzer, and calcium $(\mathrm{Ca})$ and phosphorus (P) levels were measured using an Olympus AU 2700 auto-analyzer (Beckman Coulter, California, USA). The BAP concentration was reported as the microgram per liter $(\mu \mathrm{g} / \mathrm{L})$. The reported with-in run CV was $1.9 \%$ and with-in day CV was $4.8 \%$

The data were evaluated using SPSS 17 package program. The minimum ( $\min )$, maximum $(\max )$, mean, standard deviation (SD) and standard error (SE) values were calculated and used todescribethedata. The controlgroupwasindependent, but the pre-tx and post-tx groups were dependent, so we did not apply a variance analysis. We used a one-to-one comparison and a probability value $(\mathrm{P})$ of 0.016 , based on the Bonferroni correction, with $95 \%$ confidence intervals. The pre-tx and post-tx groups were compared to the control group using the Student T-Test. The pre-tx and post-tx groups were compared to each other using the Paired Sample T-Test. Patients were also grouped according to gender, and the Mann-Whitney U Test was applied to investigate differences between genders.

\section{Results}

The descriptive values of $\mathrm{Ca}$ and BAP levels obtained for the
Table 2. The results of statistical comprehension of BAP levels.

\begin{tabular}{lcc} 
& P Level & Statistical Method \\
\hline Control group vs. Pre-tx group & $\mathrm{p}<0.001$ & Student t test \\
Control group vs. Post-tx group & $\mathrm{p}<0.001$ & Student $t$ test \\
Pre-tx group vs. Post-tx group & $\mathrm{p}=0.023$ & Paired Sample $t$ test \\
\hline
\end{tabular}

control, pre-tx and post-tx groups are shown in Table 1. The mean values and standard errors of each group are shown in Figure 1. In terms of BAP levels, there were significant differences between the control group and the pre-tx group $(\mathrm{p}<0.001)$ and between the control group and the post-tx group $(\mathrm{p}<0.001)$ (Table 2$)$. The BAP levels were lower in the post-tx group than the control group, but this difference was not statistically significant $(\mathrm{p}=0.023)$ (Fig. 1). The $\mathrm{Ca}$ and $\mathrm{P}$ levels were significantly different between the pre-tx and post-tx groups $(\mathrm{p}=0.001$ and $\mathrm{p}<0.001)$. There were no significant differences between males and females in the pretx group $(\mathrm{p}=0.83)$ or or the post-tx group $(\mathrm{p}=0.87)$.

\section{Discussion}

In conclusion, we observed that BAP levels were significantly lower in the pre-tx and post-tx groups than that in the control group. There were no any significant differences between the pre-tx and post-tx groups. These results suggest that osteoblast activity is suppressed during ESRD and that transplantation worsens osteoblastic inactivity, thus causing the bone to enter a non-dynamic state. Bone is a dynamic tissue that is continuously remodeling itself through osteoclastic bone resorption and osteoblastic bone formation. A bone biopsy is the gold standard for the diagnosis of renal osteopathology, but this method is invasive and, thus, is not preferred. Rojas et al (2003) reported that, 'Pretransplant biopsies showed different histologic abnormalities, including osteitis fibrosa, mixed and adynamic bone disease, osteomalacia, and osteoporosis. Most of the patients showed a decrease in posttransplant osteoblast surfaces and number independent

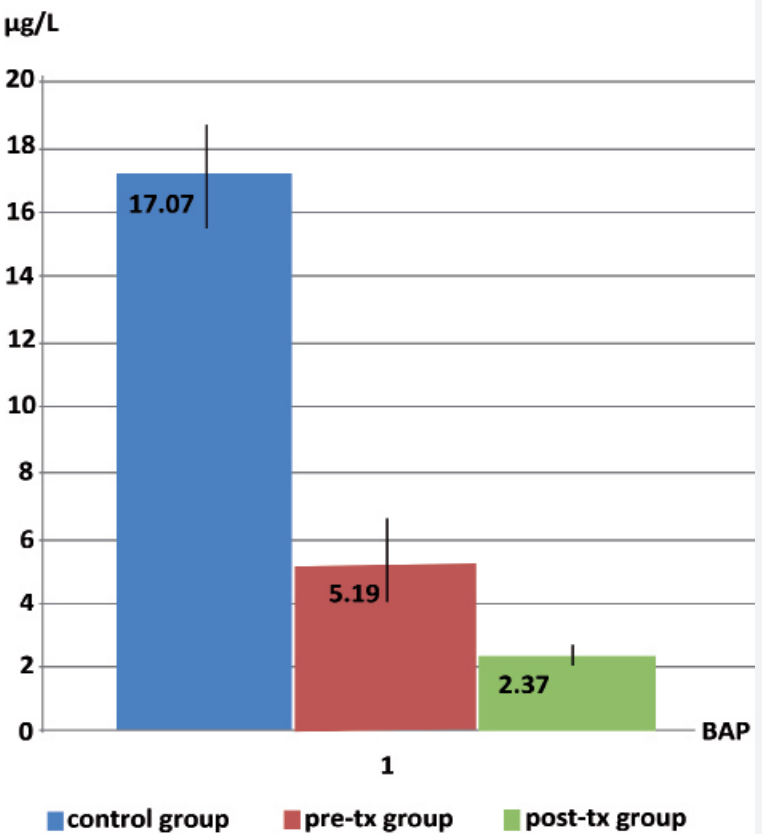

Fig. 1. Mean and standard error values of control, pre-tx and post-tx groups 
of the predominant bone disease'. These are data consistent with our findings. The measurement of bone mineral density is non-invasive and may provide useful information. Various biochemical markers have been proposed for monitoring bone metabolism; however, no single biochemical marker is available for monitoring renal osteopathology (Małyszko and Wolczyn ski, 2003). During osteoclastic bone resorption, collagen type I breakdown into fragments and released into the circulation and excreted in the urine and can be measured in the serum or urine as bone resorption markers. Pyridinoline, deoxypryridinoline, crosslinked N-terminal telopeptides of type I collagen and C-terminal telopeptides of type I collagen are markers used to evaluate bone resorption (Kusec and Smalcelj, 2004). BAP reflects an increase in bone turnover and is useful for monitoring bone formation (Malluche and Claude, 2010). However, BAP is thought to be an unpredictable marker because heterogeneous factors, such as the duration of dialysis, secondary hyperparathyroidism, aluminum osteomalacia and postoperative glucocorticoid therapy, influence bone metabolism (Rix and Andreassen, 1999). Monier-Faugere and Mahwah (2000) reported that the high doses of glucocorticoids that are given in the first few months after transplantation may be the cause of the low bone turnover that is observed at a high prevalence in renal transplantation patients. Kobayashi and Shidara (2012) and Dresher and Verduijn (2011) reported that longer dialysis duration increases BAP levels and associated with increased in mortality. Kawarazaki and Shibagaki (2012) reported that BAP levels increased at one year after transplantation. The duration of hemodialysis and ESRD inversely affect the healing time of patients with bone disease after transplantation (Jose and Weisinger, 2006; Mitterbauer and Oberbauer, 2008).

Our results may be valid for the patient group studied here and provide useful information about the early postoperative effects of renal transplantation. BAP may be a useful parameter for assessing the metabolic status of bone before and after transplantation. Many studies with longer post-operative observation periods are needed to evaluate the ability of BAP and other bone resorption markers to assess bone metabolism.

\section{Acknowledgments}

BAP kits were donated by Beckman Coulter (Istanbul, Turkey).

\section{REFERENCES}

Drechsler, C., Verduijn, M., 2011. Bone alkaline phosphatase and mortality in dialysis patients. Clin. J. Am. Soc. Nephrol. 6, 1752-1759. doi: $10.2215 /$ CJN.10091110.

Heaf, J.G., 2003. Bone disease after renal transplantation. Transplantation. 75, 315.

Jose', R., Weisinger, Rau'G, 2006. Bone disease after renal transplantation. Clin. J. Am. Soc. Nephrol. 26, 1300-1313.

Kawarazaki, H., Shibagaki, Y., 2012. Kidney transplantation restored uncoupled bone turnover in end-stage renal disease. Clin. Nephrol. 78, 10-16.

Kobayashi, I., Shidara, K., 2012. Higher serum bone alkaline phosphatase as a predictor af mortality in male hemodialysis patients. Life Sci. 30, 212-218. doi: 10.1016/j.lfs.2011.11.006.

Kusec, V., Smalcelj, R., 2004. Importance of biochemical indicators of bone turnover in patients on chronic dialysis and after kidney transplantation. Acta. Med. Croatica. 58, 51-57.

Malluche, H., Claude, M., 2010. Bone disease after renal transplantation. Natur. Rev. Nephrol. 6, 32-40. doi: 10.1038/nrneph.2009.192.

Małyszko, J., Wołczyn' ski, S., 2003. Correlations of new markers of bone formation and resorption in kidney transplant recipients. Transplantation Proc. 35, 1351-1355.

Mitterbauer, C., Oberbauer, R., 2008. Bone disease after kidney transplantation european society for organ transplantation. Transpl Int. 21, 615624. doi: $10.1111 /$ j.1432-2277.2008.00665.x.

Monier-Faugere, M.C., Mahwah, H., 2000. High prevalence of low bone and occurrence of osteomalacia after kidney transplantation. J. Am. Soc. Nephrol. 11, 1093-1099.

Rix, M., Andreassen, H., 1999. Bone mineral density and biochemical markers of bone turnover in patients with predialysis chronic renal failure. Kidney International. 56, 1084-1093.

Rojas, E., Raul, G., Carlini, R.G., 2003. The pathogenesis of osteodystrophy after renal transplantation as detected by early alterations in bone remodeling. Kidney International. 63, 1915-1923.

Sardiwal, S., Gardham, C., 2012. Bone-specific alkaline phosphatase concentrations are less variable than those of parathyroid hormone in stable hemodialysis patients. Kidney International. 82, 100-105.

Weiss, M.J., Ray, K., 1988. Structure of the human liver/bone/kidney alkaline posphatase gene. J. Biol. Chem. 263, 12002-120010. 